\title{
Generation of liver organoids by 3D co-culturing of hepatocytes, hepatic stellate cells, and liver sinusoidal endothelial cells
}

Sean C. Mulligan, $\mathrm{MS}^{1}{ }^{1}$, Wenjun Zhang, $\mathrm{PhD}^{1}$ Lester J. Smith, $\mathrm{PhD}^{2,3}$, Erika Gramelspacher, BS ${ }^{1}$, Ping Li, $\mathrm{PhD}^{1}$, Burcin Ekser, MD, $\mathrm{PhD}^{1}$

(1) Transplant Surgery, Department of Surgery, Indiana University School of Medicine; (2) 3D-Bioprinting Core, Indiana University School of Medicine; (3) Department of Radiology and Imaging Sciences, Indiana University School of Medicine

Background and Hypothesis: Recent progress with combination of chemicals in cell culture media prolongs hepatocyte $(\mathrm{HC})$ function in vitro. However, without other cells of hepatic lineage that comprise natural liver, HC alone are not suitable for the study of complex liver diseases. Hepatic stellate cells (HSC) and liver sinusoidal endothelial cells (LSEC) play vital roles in physiological and pathological liver function in situ. We hypothesized that coculturing primary $\mathrm{HC}$ with HSC and LSEC as 3D liver organoids in the same chemical conditioned media would uphold $\mathrm{HC}$ function over time, creating a better physiological 3D liver microenvironment for liver disease research.

Experimental Design: Freshly thawed primary human HCs were combined with immortalized human HSC alone, or together with immortalized human LSECs, to generate 3D liver spheroids. Spheroids were formed in HC maintenance media (HMM, Lonza) using low adhesion 96-well plates for 6 days before switching to the media with different combination of chemicals (SB31542, Forskolin, IWP2, DAPT, and LDN193189) for culturing another 14 days. Spheroids characterization, albumin secretion, mRNA transcription (CYP3A4), and histological analysis were performed for $\mathrm{HC}$ differentiation, maturation, and function.

Results: Both co-cultures of HC:HSC (2.5:1 ratio), and HC:HSC:LSEC (2.5:1:1) formed spheroids in HMM within 4 to 6 days (Fig.1A). The introduction of the different chemical-based media affected the roundness and diameter of spheroids differently (Fig.1B). In the presence of all 5 chemicals $(5 \mathrm{C}), \mathrm{HC}$ function was better maintained up to 21 days in HC:HSC:LSEC spheroids, measured by albumin, CYP3A4, and CK-19 secretion (Fig.1C). Surprisingly, 5C-based media significantly upregulated the expression of CK-19, which is one of the markers for cholangiocytes and liver precursor cells.

Conclusion and Potential Impact: 3D liver organoids composed of HC, HSC, and LSEC would create a niche environment mimicking the in vivo condition. Optimization of complex hepatic spheroids, as well as optimization of complex hepatic spheroid culturing media, would allow for the generation of a unique model for studying complex liver diseases. 


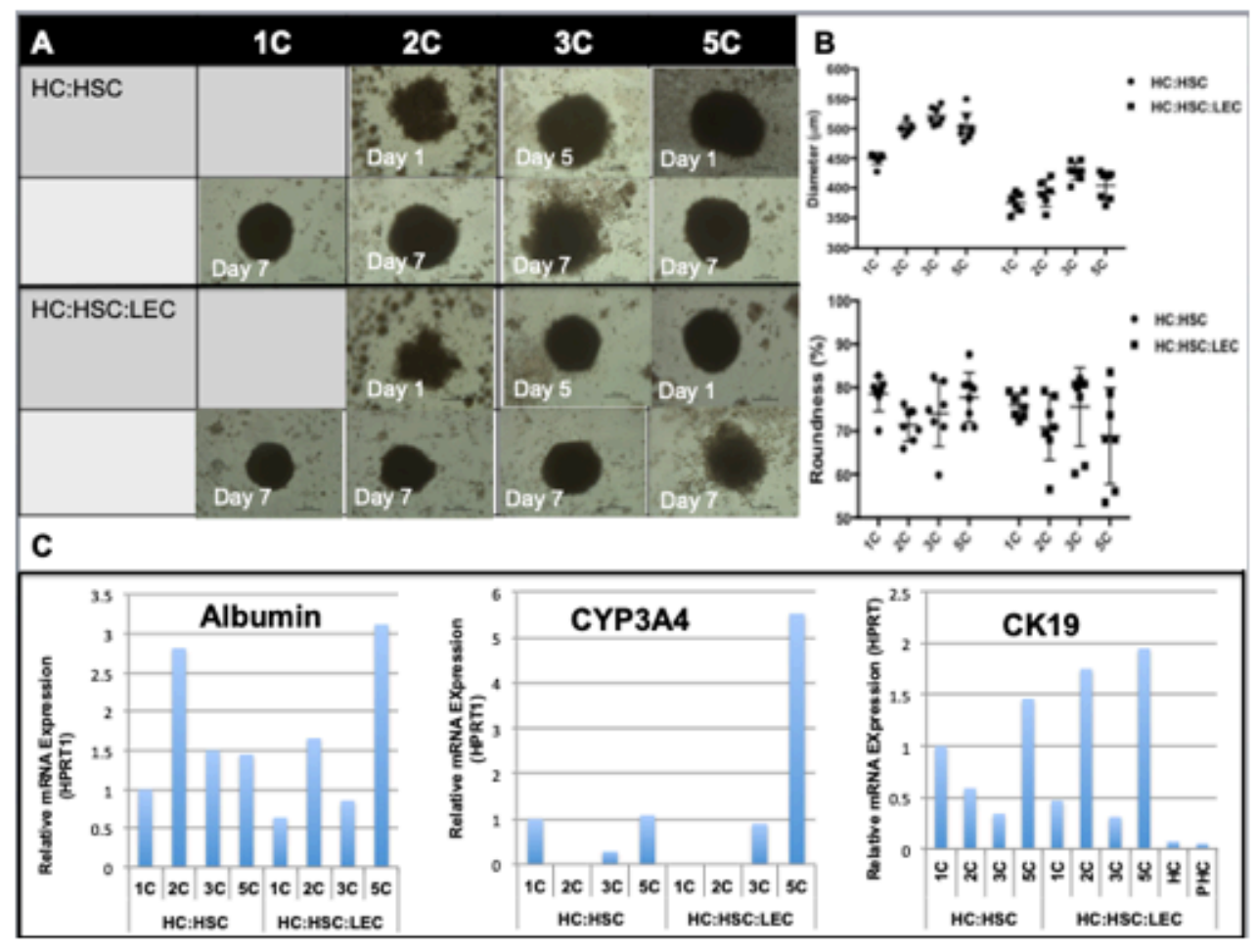

Figure 1: A. Images of spheroid formations in each of the spheroid type and chemical conditions. 5C $=$ SB43, Forskolin, DAPT, IWP2, LDN1931. 3C= SB43, Forskolin, DAPT. 2C= SB43, Forskolin. 1C= SB43. B. Spheroid diameter (top) and roundness (bottom) in different chemical conditions and cell types. Cell combinations were characterized by Regenova Bioprinter (Cyfuse Biomedical, Japan). C. Real-Time qRT-PCR analysis for albumin, CYP3A4, and CK-19 expression in indicated spheroid type and chemical conditions after 14 days of culture in chemical conditioned media (total 21 days).

\section{Funding Support:}

- Short-Term Training Program in Biomedical Sciences Grant. T35 HL 110854

- Office of The Director, National Institutes of Health of the National Institutes of Health under Award Number S100D023595

- Board of Directors of the Indiana University Health Values Fund for Research Award (VFR-457-Ekser). 